\title{
Systematic Review: National Notifiable Infectious Disease Surveillance System in China
}

\section{Liping Wang1, Xiang Ren ${ }^{1}, 2$, Benjamin J. Cowling ${ }^{2}$, Lingjia Zeng1, Mengjie Geng ${ }^{1}$, Peng Wu2, Zhongjie Li ${ }^{1}$, Hongjie Yu ${ }^{3}$, George Gao 1}

${ }^{1}$ Chinese Center for Disease Control and Prevention, Beijing, Beijing, China, ${ }^{2}$ The University of Hong Kong, Hong Kong, Hong Kong, China, ${ }^{3}$ Fudan University, Shanghai, China

\section{Objective}

We aimed to review the development and changes of National Notifiable Disease Surveillance System (NNDSS) from 1950 to 2013, and to analyze and summarize the changes in regulations and public health surveillance practices in China.

\section{Introduction}

Infectious disease was the second most common cause of death in 1949, and the epidemic situation of infectious diseases was so severe that the Chinese government made major investments to the control and prevention of infectious diseases. During the past 60 years the development of the notifiable disease surveillance system in China has experienced 3 phases, including germination stage, development stage, improvement and consolidation stage [1]. As the quality of infectious diseases surveillance has been improved stepwisely, the national morbidity of class A and B notifiable disease decreased from 7157.5 per 100,000 in 1970 to 225.8 per 100,000 in 2013 , and the mortality decreased from 56.0 per 100,000 in 1959 to 1.2 per 100,000 in 2013 [2].

\section{Methods}

Research articles, online reports and grey literature from January 1950 to February 2013 relevant to disease surveillance in China were searched in databases including PubMed, China National Knowledge Infrastructure (CNKI), and Wanfang Data. Retrieved articles were screened by inclusion criteria of containing the infectious diseases prevention and control, related laws and regulations, and development of surveillance system.

\section{Results}

In the systematic review, 20 articles were retrieved from PubMed, 1129 articles from CNKI, 480 articles from WanFang database, after abstract screening and eliminating overlaps, 73 articles were included, including 10 English articles and 63 Chinese articles.

\section{Laws and regulations on notifiable diseases in China}

\begin{abstract}
Administrative Measures for Infectious Diseases Control was issued in 1955 to deal with 18 diseases (classes A and B) for their notification, monitoring, reporting and treatment. In 1956, 7 more infectious diseases were added into class B infectious diseases. Regulation on the Administration of Acute Infectious Diseases was issued in 1978, infectious diseases in class A and B including suspected cases must be reported within specific time respectively. The Law of the People's Republic of China on the Prevention and Treatment of Infectious Diseases, the first infectious disease law in China, was issued in 1989 and revised in 2004. The number of notifiable infectious diseases was increased to 35, including 2 class A, 21 class B and 12 class C notifiable diseases in 1989. The 2004 revised version contained total 37 notifiable diseases and clarified infectious disease prevention, epidemic situation report, notification and release, epidemic control, medical treatment, supervision and management, logistic measures, legal responsibility and supplementary provisions.
\end{abstract}

\section{The organization of notifiable disease surveillance and management}

In 1950s, the Government Administration Council approved the bill of the establishment of Health Epidemic Prevention Stations (HEPS) nationwide. Chinese Academy of Medical Sciences (CAMS) was established in 1956, and the Chinese Academy of Preventive Medicine (CAPM) was established in 1986, which was in charge of the national infectious disease surveillance data collection, management, analysis and feedback. In 2002, the CAPM officially changed its name to the Chinese Center for Disease Control and Prevention (CDC), so did all levels of health epidemic prevention station. As mentioned in the Law of the People's Republic of China on the Prevention and Treatment of Infectious Diseases, CDCs at all levels are responsible for infectious disease

SDS Annual Conference Proceedings 2019. This is an Open Access article distributed under the terms of the Creative Commons AttributionNoncommercial 4.0 Unported License (http://creativecommons.org/licenses/by-nc/3.0/), permitting all non-commercial use, distribution, and reproduction in any medium, provided the original work is properly cited. 
surveillance, prediction, epidemiological investigation, epidemic reporting and other prevention and control. In addition, the law clarified the establishment of infectious disease surveillance system, the specific duties and tasks of the administrative department of public health and healthcare technology institutions in the infectious disease surveillance (Figure 1).

\section{Notifiable diseases diagnostic criteria}

Notifiable Diseases Diagnostic Criteria (Trial Edition) was issued in 1990 and revised in 2004. Diagnostic Criteria defined suspected case, probable case and confirmed case. Suspected case mainly based on clinical symptoms and signs; probable case was the suspected cases with hemogram blood test. Confirmed case was based on blood test results and pathogen specific antigen or antibody test results, e.g. Ig G, Ig M or virus tested positive among suspected or probable cases.

\section{Reporting method of notifiable diseases surveillance system}

During 1950 to 1985 , monthly collection of reports was delivered by post mail level by level hierarchically (from county HEPSs to prefectural or city HEPSs, then to provincial HEPSs and eventually to CAPM). The notifiable infectious diseases reporting network covered the whole country firmly launched at the mid-1960s.

In 1986, the prototype of electronic reporting was sprout. Over 200 network nodes achieved electronic submission of the national notifiable infectious diseases monthly report by post-delivery, some provinces even had adopted more efficient reporting means by e-mail autonomously. During 1986 to 2003, different reporting cards are used for collecting class A, B, and C infectious diseases respectively. At the beginning of each year, the provincial HEPSs updated the population census data and the administrative changes. At the end of each year, the provincial HEPSs reported detailed age-gender and occupation specific diseases morbidity and mortality data, as well as amended monthly reports for delay or missing, to CAPM.

The internet-based timely reporting system was officially launched in 2004. China CDC built the notifiable infectious diseases and emergent public health event reporting system that covered all hospitals and medical institutes nationwide, which collected individual case data with unified reporting card. By 2013 the system with over 70,000 reporting units covered 100\% county and above level CDCs, $98 \%$ of county and above level medical institutions, and $94 \%$ of township level healthcare units.

\section{Conclusions}

Monthly reporting was replaced by real-time reporting, and the weekly, monthly and yearly cumulative incidence and death was replaced by individual case reporting. The hierarchical reporting structure, were changed to reporting directly to national data center. The notifiable disease surveillance system network has been expanded, the diagnosis capacity and criteria, surveillance data report methods and sensitivity have been improved gradually. The notifiable disease surveillance system optimized step by step with internet-based timely reporting technology and direct filling infectious disease case information from healthcare facilities.

\section{Acknowledgement}

The views expressed are those of the authors and do not necessarily represent the policy of the Chinese Center for Disease Control and Prevention.

\section{References}

1. Cheng M. 2005. etc. The history and development trend of disease surveillance in China. Dis Surveill. 20(3), 113-14.

2. The national health and family planning commission of the People's Republic of China. The national epidemic situation of notifiable diseases in 2012. 


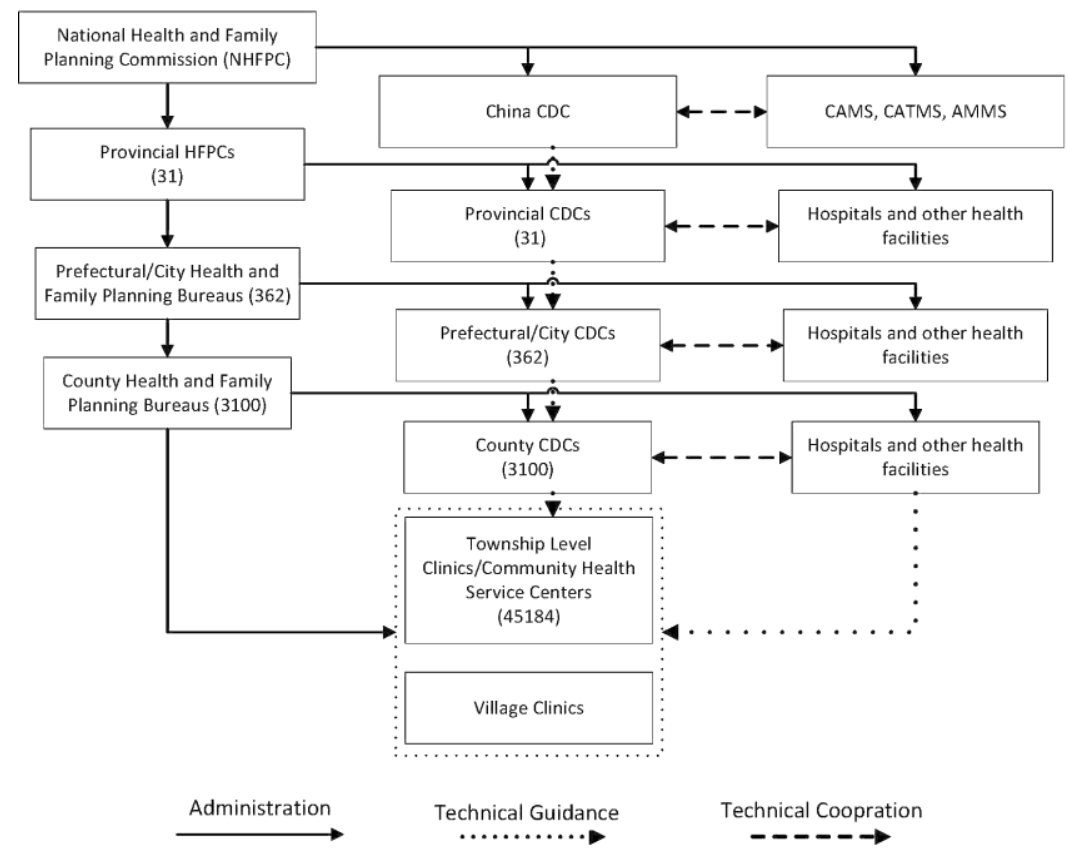

Figure 1 Organization chart of notifiable diseases surveillance system 